\title{
Evaluation of various mineralization methods and measurement techniques for trace element analysis of plant materials
}

\author{
E. Clurdová1,*, J. Száková², D. Miholová², O. Mestek ${ }^{1}$ and M. Suchánek ${ }^{1}$ \\ ${ }^{1}$ Institute of Chemical Technology, Technická 5, 16628 Prague, Czech Republic \\ ${ }^{2}$ Czech University of Agriculture, Faculty of Agronomy, 16521 Prague, Czech Republic
}

\begin{abstract}
Three decomposition methods (pressurised microwave digestion, classical dry ashing and dry ashing in a mixture of oxidising gases) for predominantly plant samples, and three measurement techniques (ICP-MS, FAAS, ETAAS) for the determination of $\mathrm{Cd}, \mathrm{Cu}, \mathrm{Pb}$ and $\mathrm{Zn}$ were compared. As confirmed by the statistical evaluation, no significant differences between analytical methods were found.
\end{abstract}

Key words. Plant material - mineralization -ICP-MS - FAAS - ETAAS - Cd - Cu - Pb - Zn.

\section{Introduction}

Because of its multi-element capability, high dynamic range and high sensitivity, inductively coupled plasma - mass spectrometry (ICP-MS) becomes one of the most promising analytical method for the determination of trace elements in biological and environmental materials. However, a quality assurance (QA) of data produced is necessary for a real validation of the work in any analytical laboratory. From this point of view, a regular use of certified reference materials (CRMs) and the participation in interlaboratory comparison exercises may be first important parts of a QA system in the individual laboratory.

We discuss below the analytical data for cadmium, copper, lead and zinc, which are elements of our main interest. The data were those obtained by ICP-MS in the digests of the samples: CRM 12-02-01 (Bovine liver), CRM 12-02-03 (Lucerne) and six plant samples from an international comparison run. For aliquots of the same digests, ICP - MS data are compared with those obtained by atomic absorption spectrometry (AAS), well known technique which may be considered as a reference. Moreover, all the data obtained were compared with certified values for CRMs, or with recommended values in the case of samples from intercomparison exercise.

\section{Experimental}

\section{Samples}

a) six plant materials (Barley, Carnation, Lucerne, Maize, Melon and Oil palm leaves) from the framework of International Plant-Analytical Exchange (IPE 1996.3) organised by WEPAL, Wageningen (The Netherlands) [1];

b) Certified reference materials CRM 12-02-03 (Lucerne), prepared by the Institute of Radioecology and Applied
Nuclear Techniques, Kos lice, CS [2] and CRM 12-02-01 (Bovine Liver) by the Czechoslovak Institute of Metrology, Bratislava, CS [3].

\section{Mineralization}

Three various sample decomposition techniques were applied as follows:

1. Wet ashing under pressure with focused microwave heating (Plazmatronika BM-1S, Poland). Samples (approximately $0.5 \mathrm{~g}$ ) were weighed accurately in PTFE digestion bombs and $3 \mathrm{~mL}$ conc. $\mathrm{HNO}_{3}$ (Suprapur, Merck) added. Digestion was then performed for $3 \mathrm{~min}$ at $210 \mathrm{~W}$ followed by $7 \mathrm{~min}$ at $270 \mathrm{~W}$ (max. power $300 \mathrm{~W}$ ). After cooling for $10 \mathrm{~min}$, the digests were quantitatively transferred into $50 \mathrm{~mL}$ volumetric flasks and filled to volume with deionnised water.

2. Classical dry ashing at $500{ }^{\circ} \mathrm{C}$ in a muffle furnace (open system) [4]. Samples (1.0 g) were weighed in $50 \mathrm{~mL}$ quartz beakers, charred on a hot plate with stepwise increasing temperature up to $350{ }^{\circ} \mathrm{C}$ for 4 hours, and finally ashed in a muffle furnace at $500{ }^{\circ} \mathrm{C}$ for 16 hours. After cooling, ashes were dissolved in $20 \mathrm{~mL}$ of $1.5 \% \mathrm{HNO}_{3}$.

3. Dry ashing in a mixture of oxidising gases at $400{ }^{\circ} \mathrm{C}$ for 14 hours in a dry mode mineralizer APION (Tessek, Czech Republic) [5]. In this particular device, the mineralization process is achieved by the combination of dry ashing between 300 and $400{ }^{\circ} \mathrm{C}$, and a strong oxidation in a mixture of superoxidative gases $\left(\mathrm{O}_{2}+\mathrm{O}_{3}+\mathrm{NO}_{x}\right)$ prepared in situ in the instrument.

Sample weights and dissolving of ashes is similar as in the previous procedure.

At least duplicate analysis (decomposition and measurement) were accomplished for each sample. A set of blank solutions ( $25 \%$ of all samples) was prepared using the same protocol which was applied for real samples. 


\section{Apparatus}

The main part of measurements was performed using an Elan 6000 (Perkin-Elmer Sciex, USA) ICP - mass spectrometer equipped by a Gilson 212 peristaltic pump, a crossflow nebulizer and a Scott spray chamber. The operating conditions used are summarised in table I. The following isotopes were used for the signal evaluation: ${ }^{65} \mathrm{Cu} ;{ }^{66} \mathrm{Zn}$; ${ }^{111} \mathrm{Cd}$ and the sum of ${ }^{206} \mathrm{~Pb},{ }^{207} \mathrm{~Pb}$ and ${ }^{208} \mathrm{~Pb}$ for $\mathrm{Pb}$ measurements. In order to eliminate errors caused by differences in lead isotope composition of samples of various origin, the sum of mentioned $\mathrm{Pb}$ intensities was evaluated as an analytical signal. ${ }^{115} \mathrm{In}$ and ${ }^{209} \mathrm{Bi}$ (both at $100 \mu \mathrm{g} \mathrm{L} \mathrm{L}^{-1}$ ) were used as internal standards. Before measurements, the digests were diluted to obtain similar concentration of dissolved salts in solutions for all the decomposition procedures.

SpectrAA-400 (Varian, Australia) atomic absorption spectrometer was used in flame mode with a quartz concentrator tube (STAT) for the determination of $\mathrm{Zn}$ and $\mathrm{Cu}$ or in flameless mode using a graphite furnace atomizer (GTA-96) for the determination of $\mathrm{Cd}$ and $\mathrm{Pb}$.

\section{Statistics}

Statistical evaluation of the analytical data was performed using non-parametric and robust Wilcoxon T - test [6] which was applied for a comparison of decomposition methods used (significance level $\alpha=0.05$ ). A linear regression was applied for the comparison of measurement techniques (ICP$\mathrm{MS}=a+b \cdot \mathrm{AAS})$. Z-scores were applied for the accuracy evaluation of the measured data using following formulas:

$$
\begin{gathered}
Z=\frac{Y-Y_{(M .)}}{M A D} \text { for IPE samples and } \\
Z=\frac{Y-Y_{(C V .)}}{\bar{\sigma}} \text { for reference materials, }
\end{gathered}
$$

where $Y_{(\mathrm{M})}$ is median of IPE values, MAD is median of absolute deviations, $Y_{(\mathrm{CV})}$ is certified value and $\bar{\sigma}$ is combined uncertainty of this certified value. The usual way of the $Z$ score classification as $|Z| \leq 2$ - satisfactory, $2<|Z|<3$ questionable, and $|Z| \geq 3$ - unsatisfactory was used in the evaluation of data obtained.

\section{Results and discussion}

Data for evaluation (Tabs. II and III) were obtained by repeated measurements for each analyte for the three different digestion procedures. Because of a small amount of samples from IPE test, only two replicates of decomposition have been performed. All sample solutions were analysed by both ICP-MS and AAS techniques. Consequently, the whole evaluated set of samples displayed 48 values.

Linear regression (Figs. 1-3) shows a good accordance of both measurement methods for cadmium, lead and copper ( $a$ was not differed from zero and $b$ from one). In the case of zinc, intercept $a$ differs significantly from zero (Fig. 4). Leverage point caused by CRM 12-02-01 data (Bovine liver) is evident. The contents of $\mathrm{Zn}$ found in bovine liver by ICPMS are in this case higher as compared to both AAS and certified values. After exclusion of values found for this sample (the only one of animal origin in our study) the $a$ intercept is not significantly different from zero (Fig. 5).
Table I. ICP - MS operating conditions.

\begin{tabular}{lc}
\hline Sweeps/Reading & 5 \\
Replicates & 3 \\
Dwell time (ms) & 200 \\
Gas flow rates(L/min) & \\
$\quad$ coolant & 17.0 \\
$\quad$ auxiliary & 1.2 \\
$\quad$ nebulizer & 0.775 \\
Forward power(W) & 1000 \\
Sample uptake rate(mL/min) & 1.0 \\
Integration time $(\mathrm{ms})$ & 1000 \\
\hline
\end{tabular}

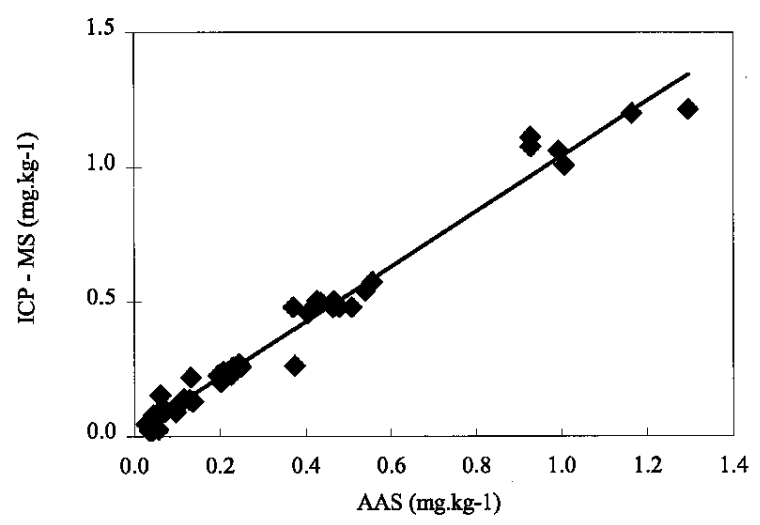

Fig. 1. Correlation plot of the cadmium concentration in the digests analysed by AAS $(x)$ and ICP-MS $(y), r=0.989 ; y=(0.015 \pm$ $0.020)+(1.025 \pm 0.045) \cdot x$.

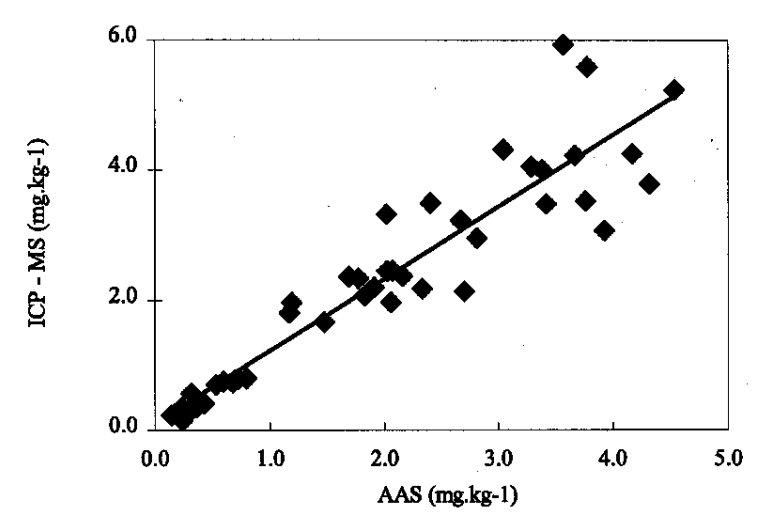

Fig. 2. Correlation plot of the lead concentrations in the digests analysed by AAS $(x)$ and ICP-MS $(y), r=0.993 ; y=(0.30 \pm$ $0.40)+(0.982 \pm 0.036) \cdot x$.

As can be concluded from the Wilcoxon T-test for $\mathrm{Cd}$ and $\mathrm{Cu}$, no significant differences are observed at the significance level $\alpha=0.05$ among all the decomposition techniques used. For $\mathrm{Pb}$, significantly lower results were obtained for classical dry ashing and APION decomposition if compared to microwave decomposition followed by AAS measurement. This difference was not found for ICP-MS results. However, as can be seen in figure 2, the variability of $\mathrm{Pb}$ data is in general relatively high. For $\mathrm{Zn}$, the lowest results were observed for digests obtained by classical dry 


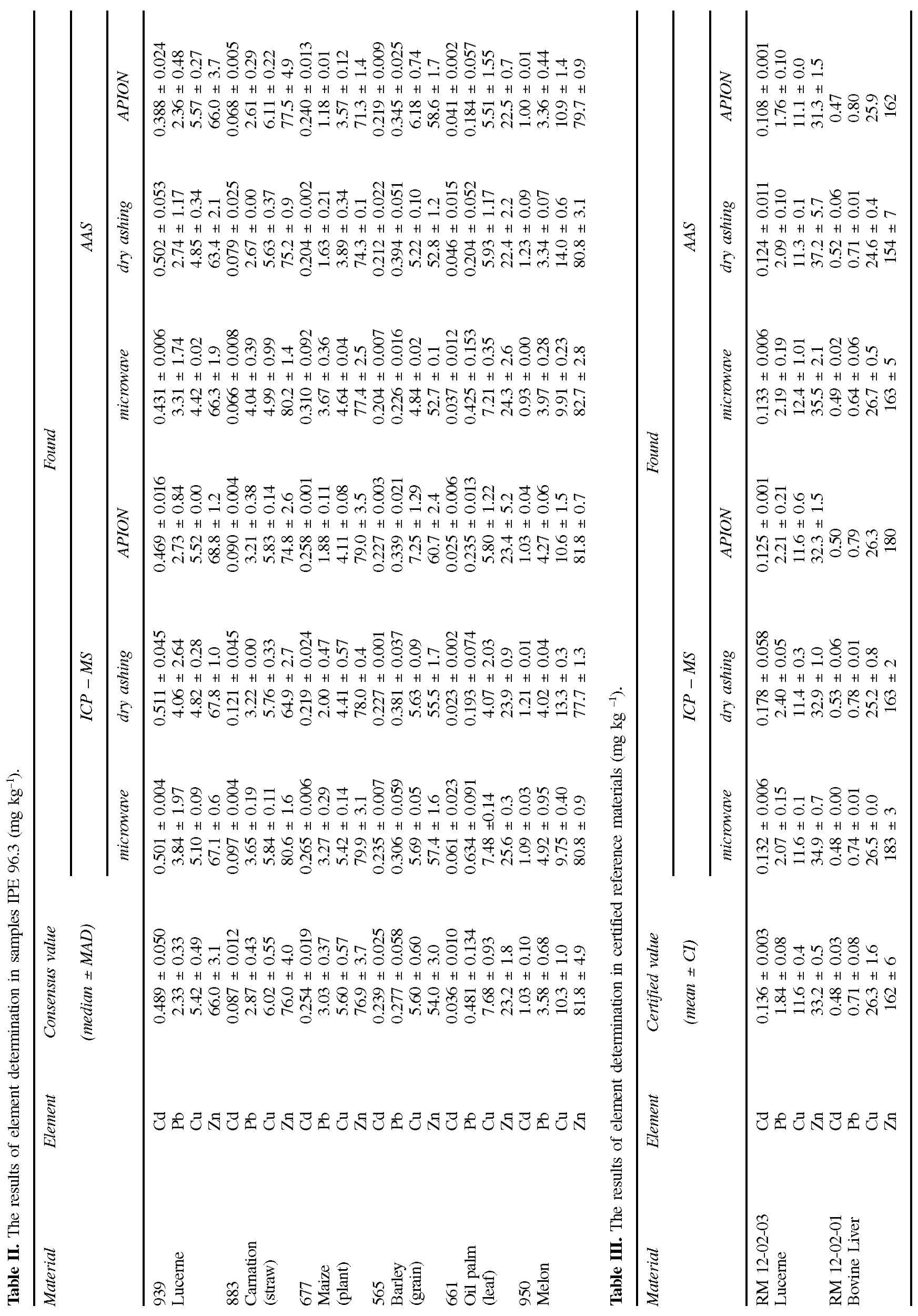




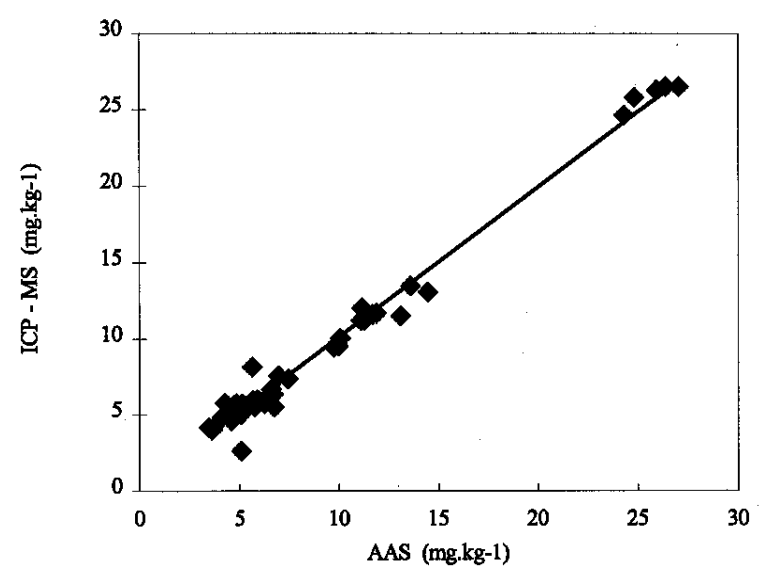

Fig. 3. Correlation plot of the copper concentrations in the digests analysed by AAS $(x)$ and ICP-MS $(y), r=0.939 ; y=(0.11 \pm$ $0.28)+(1.106 \pm 0.121) \cdot x$.

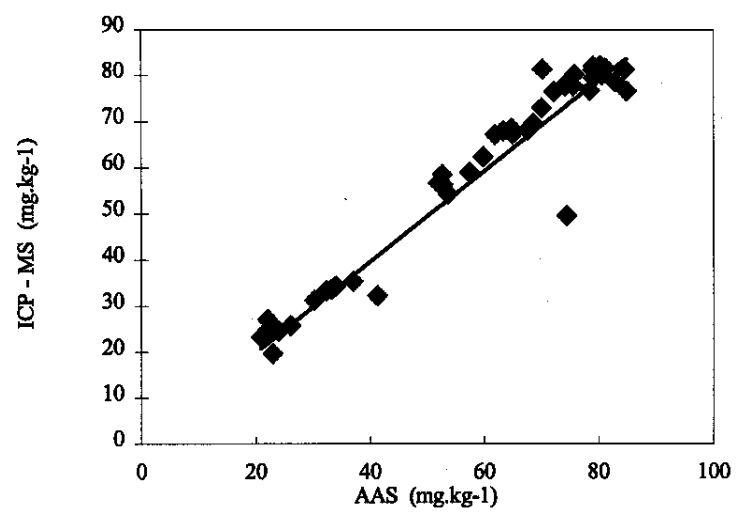

Fig. 5. Correlation plot of the zinc concentrations in the digests analysed by AAS $(x)$ and ICP-MS $(y), r=0.968 ; y=(2 \pm 5)+$ $(0.978 \pm 0.082)$. $x$. Data for RM 12-02-01 Bovine Liver (certif. value $162 \pm 7 \mathrm{mg} \mathrm{Zn} \mathrm{kg}^{-1}$ ) excluded from the evaluation.

ashing, higher data after APION decomposition and the highest ones after the microwave heating if ICP-MS is used for measurement. As concerns AAS data, no significant differences were found after the APION decomposition and microwave digestion but some lower results appeared after classical dry ashing.

The accuracy of the results was evaluated using $Z$-scores: the obtained data are summarised in figures 6 and 7. A comparison with certified or recommended values leads to conclusion that $\mathrm{Pb}$ values obtained by ICP-MS technique are slightly higher. This finding is similar to conclusions given in [7] for contents of $\mathrm{Pb}$ in sediments. Outlying values for $\mathrm{Zn}$-content in the animal sample (bovine liver) found by ICP-MS are higher than certified one. Similarly higher results for $\mathrm{Zn}$ content in animal sample IAEA MAM-2 (Mussel) published by Ebdon et al. [8] were explained by possible polyatomic interference (e.g. $\mathrm{HPO}_{2}{ }^{+}$) at $m / z=64$. Since measurements in this work proceed at $\mathrm{m} / z=66$, this explanation cannot be used here.

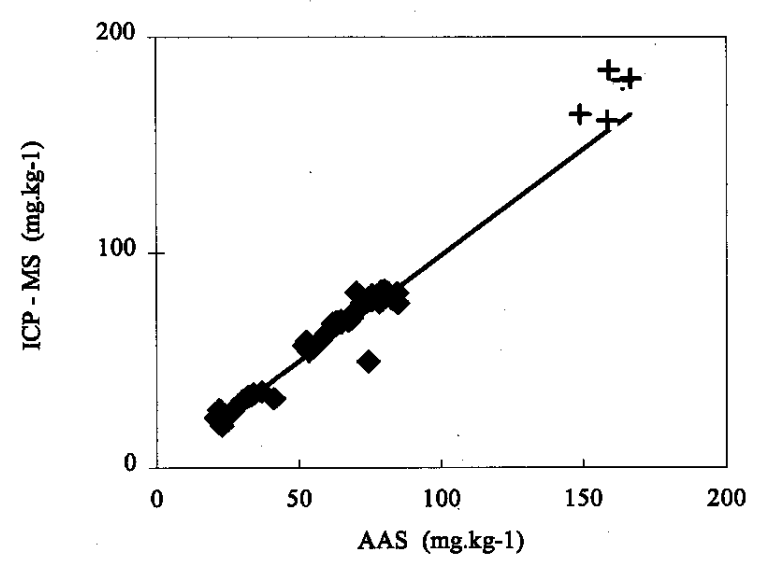

Fig. 4. Correlation plot of the zinc concentrations in the digests analysed by AAS $(x)$ and ICP-MS $(y), r=0.988 ; y=(-4.23 \pm$ $3.99)+(1.094 \pm 0.051) x$; data for bovine liver RM marked by + .

Loss of analytes as $\mathrm{Pb}$ and $\mathrm{Zn}$ during dry ashing procedure without HF-step might be provoked by their retention by silica from plant matrix [9]. When sample of oil palm leaves was decomposed by dry ashing with following dissolution of ashes in $\mathrm{HNO}_{3}$ - $\mathrm{HF}$ mixture, evaporation and dissolution of residue in $1.5 \% \mathrm{HNO}_{3}$, accurate results of $\mathrm{Pb}$ were obtained by the AAS technique (results of Laboratory 214 in [1]).

\section{Conclusion}

Both measurement methods provide comparable results with a good agreement. As regards of our analytical data from certified/recommended values it can be concluded that results obtained are affected in greater extent by the decomposition procedure than by measurement techniques. In comparison with ICP-MS it seems that AAS is more influenced by resulting matrix from various decomposition procedures particularly if analysed material contains silicates. This conclusion shows once again that a suitable digestion procedure is of paramount importance.

\section{Acknowledgement}

The financial support of the Ministry of Environment of the Czech Republic (project No. VAV/340/2/96) is gratefully acknowledged.

\section{References}

1. Houba, J. V. G. et al. IPE 1996, Bimonthly Report 96.3., Department of Soil Science and Plant Nutrition, Wageningen, The Netherlands, 1996.

2. Bartha, $\mathrm{S}^{\prime}$; Kalinc ḱk, M.; Kladeková, D. Report on Intercomparison P - ALFALFA for the Determination of Essential and Toxic Elements in Lucerne, Kos Ice, CS, 1989.

3. Kuc era, J.; Mader, P.; Miholová, D.; Cibulka, J.; Poláková, M.; Kordík, D.; Fresenius, J. Anal. Chem. 1990, 338, 66.

4. Mader, P.; Kuckra, J.; Cibulka, J.; Miholová, D. Chem. Listy. 1989, 83, 765.

5. Puschel, P.; Formánek, Z.; Vlasáková, A.; Stuchlíková, A. Proc. X. CANAS, 1988, Sept. 5 - 9. 


\section{Original articles}

Cd

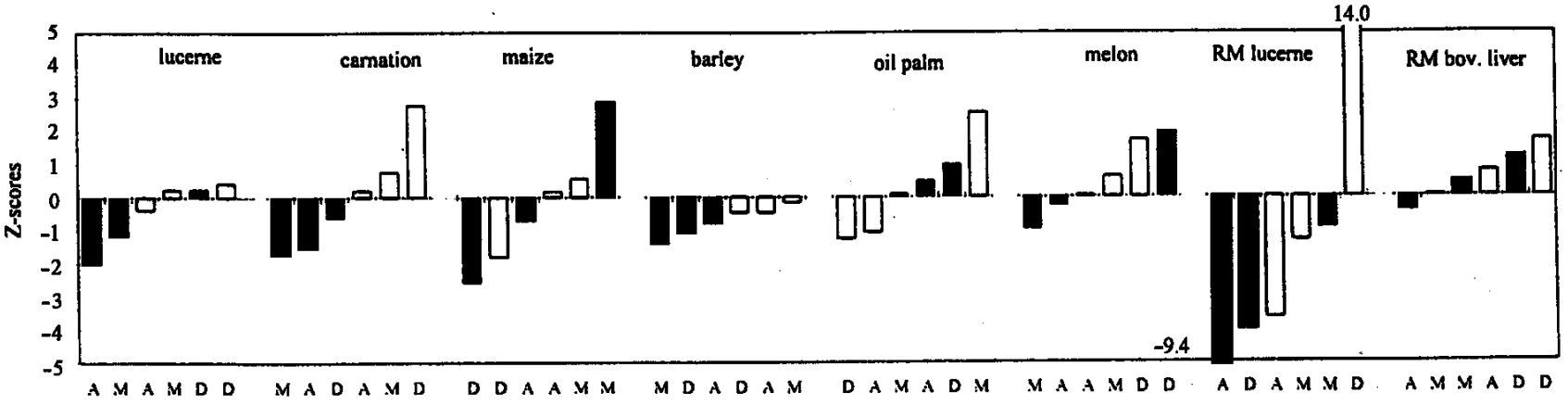

$\mathbf{P b}$

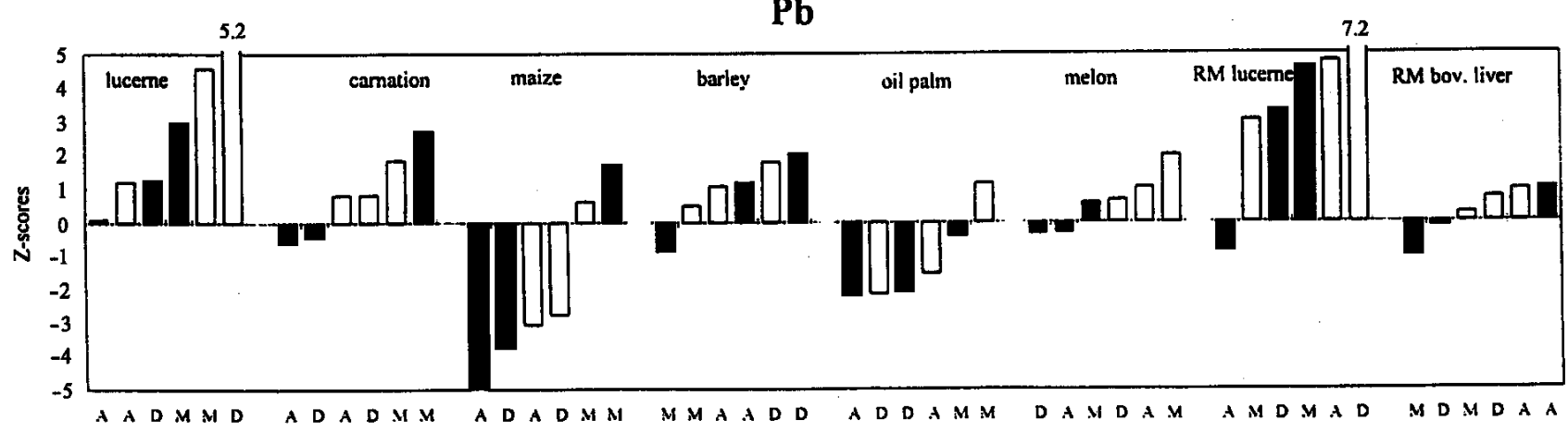

Fig. 6. Comparison of the analytical data for $\mathrm{Cd}$ and $\mathrm{Pb}$ using $\mathrm{Z}$-scores ( $\boldsymbol{\square} \ldots$ AAS, $\square$... ICP-MS, M ...microwave decomposition, D ...classical dry ashing, A ...dry ashing in APION).
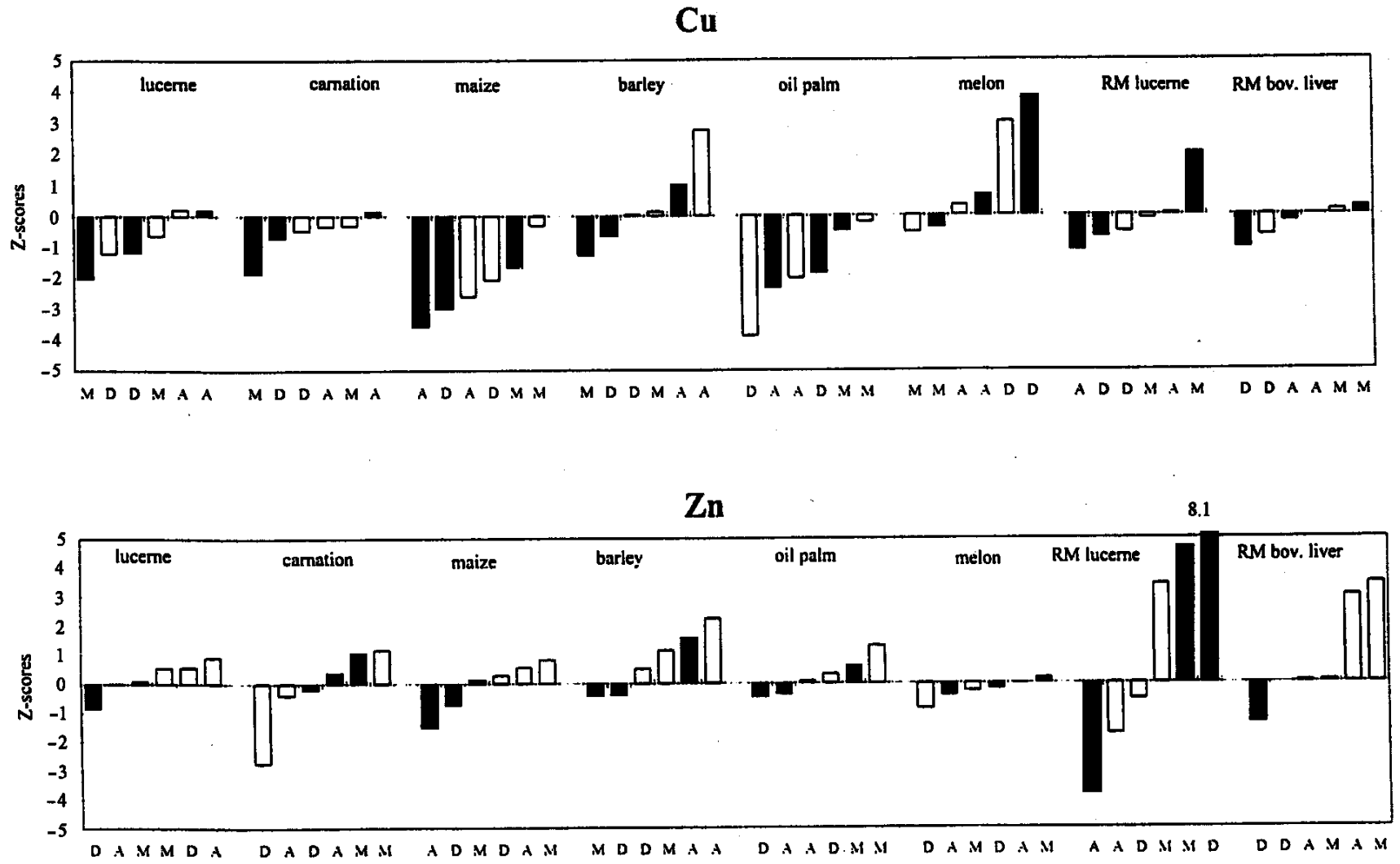

Fig. 7. Comparison of the analytical data for $\mathrm{Cu}$ and $\mathrm{Zn}$ using $\mathrm{Z}$-scores ( $\mathbf{\square} \ldots$ AAS, $\square$... ICP-MS, M ...microwave decomposition, D ...classical dry ashing, A ...dry ashing in APION). 
6. Massarrt, D. L.; Vandeginste, B. G. M.; Deming, S. N.; Michotte, Y.; Kaufman, L. Chemometrics, Elsevier, Amsterdam, 1988; p 57.

7. Jones, B. R. in: Holland, G. (Ed.) Recent Advances in Plasma Source Mass Spectrometry, Durham, UK, 1994; p 127.
8. Ebdon, L.; Fisher, A. S.; Worsfold, P. J.; Crews, H.; Baxter, M. J. Anal. At. Spectrom 1993, 8, 691.

9. Hoenig, M.; Vanderstappen, R. Analusis 1978, 6, 312. 